\title{
Polypyrrole Coated PET Fabrics for Thermal Applications
}

\author{
Amelia Carolina Sparavigna ${ }^{1}$, Luca Florio ${ }^{2}$, Jamshid Avloni ${ }^{3}$, Arthur Henn ${ }^{4}$ \\ ${ }^{1}$ Physics Department, Politecnico di Torino, Torino, Italy; ${ }^{2}$ Laboratorio Tessili Innovativi, Biella, Italy; ${ }^{3}$ Eeonyx Corporation, Pinole, \\ USA; ${ }^{4}$ Marktek Inc., Chesterfield, USA. \\ Email: amelia.sparavigna@polito.it
}

Received July $7^{\text {th }}, 2010$; revised July $26^{\text {th }}, 2010$; accepted July $27^{\text {th }}, 2010$.

\begin{abstract}
Polypyrrole can be chemically synthesized on PET fabrics, giving rise to textiles with high electric conductivity. These textiles are suitable for several applications from antistatic films to electromagnetic interference shielding devices. Here we discuss the thermal-electric performance and the heat generation of polypyrrole coated PET fabric samples, previously studied because of their electric conductivity and electromagnetic interference shielding effectiveness. The measured Seebeck effect is comparable with that of metallic thermocouples. Since polypyrrole shows extremely low thermal diffusivities regardless of the electrical conductivity, the low thermal conductivity gives significant advantage to the thermoelectric figure-of-merit ZT, comparable with that of some traditional inorganic thermoelectric materials. The heat generation is also investigated for possible heating textile devices. The results confirm polypyrrole as a promising material for thermal electric applications due to its easy preparation in low cost processing.
\end{abstract}

Keywords: Conductive Polymers, Polypyrrole, Thermoelectric Materials

\section{Introduction}

In the late 1970s, MacDiarmid, Heeger and Shirakawa discovered how to get polymers conducting electricity. ${ }^{1}$ The first material becoming an intrinsically conducting polymer (ICP) was polyacetylene, after a doping with iodine. The announcement of this discovery quickly reverberated around scientific community, and intensity of the research for other conducing polymers magnified dramatically [1-3]. A new generation of polymers was then developed, exhibiting the electrical and optical properties of metals or semiconductors, at the same time retaining the attractive mechanical properties and processing advantages of polymers.

Intrinsically conducting polymers were immediately seen as a new route to mimic metallic conductivity, besides the well-know approach to insert conductive fillers into an inherently insulating resin, or to coat a plastic substrate with a conductive metal solution [4]. In this manner, conductive fibers can be prepared to obtain conductive fabrics, or, fabrics already produced can be metalized with a conductive coating. By the way, let us

\footnotetext{
${ }^{1}$ The Nobel Prize in Chemistry 2000 was awarded jointly to Alan J. Heeger, Alan G. MacDiarmid and Hideki Shirakawa "for the discovery and development of conductive polymers".
}

note that metal coated textiles remain fundamental materials, because they generally show a high electromagnetic interference shielding effectiveness (EMI-SE) [5,6].

In fact, intrinsically conducting polymeric materials can be used to obtain rather innovative textiles [6]. These textiles are able to absorb as well as reflect electromagnetic waves, and then can exhibit certain advantages over metallic materials. Actually, the most prominent ICPs in EMI-SE are polypyrrole and polyaniline, where electrical conductivity can have values comparable to those observed for poorly conducting metals and alloy [5-8].

Among the first commercial products incorporating polypyrrole there was Contex ${ }^{\circledR}$, a conductive textile product originally manufactured by Milliken [9], starting around 1990, and now produced by Eeonyx Corp., as EeonTex ${ }^{\mathrm{TM}}$. An early application, involving the coating of polyester fibers with polypyrrole (PPy) was the creation of an antistatic fabric. Here, we will show the results of measurements of thermal electric properties of the PPy-coated EeonTex. In particular, we deduce its Seebeck coefficient, which turns out to be comparable with that of metal thermocouple materials. Because polypyrrole shows extremely low thermal diffusivity, the value of the figure-of-merit turns out to be of the same 
order of magnitude of some traditional inorganic thermoelectric materials [10].

Thermoelectric effects in ICPs are deserving a special attention, due to the considerable effort actually paid to have thermoelectric effects from low-weight but highreliable materials. The reason is that the thermoelectric cooling is a good strategy in semiconductor electronics operating at high frequencies, where the thermal management becomes crucial. Let us remember that, according to Kelvin relations between Seebeck and Peltier coefficients, an evaluation of the Seebeck effect allows estimating the cooling power of materials.

The target of this paper is then in investigating the thermoelectric effects in PPy coated samples, which have been previously studied from the point of view of their electric conductivity and electromagnetic interference shielding effectiveness $[5,6]$, We will also discuss the heat generation obtained from PPy coated fabrics, for suitable applications in textile heating systems. Polypyrrole is in fact one of ICPs very promising for wide thermal electric applications because of its easy preparation with a low cost processing.

\section{Samples Preparation}

A PPy coated textile could represent a possible solution for heating and cooling and for temperature monitoring. As previously told, one of the first commercial textile products incorporating conductive polypyrrole was the Contex conductive textile. This textile evolved in a new material, with a modified PPy coating, more conductive and thermally stable. While imparting electrical conductivity and a dark color to the substrates, the PPy coating process barely affects the strength, drape, flexibility, and porosity of the starting substrates.

For the measurements discussed in this paper, we used an EeonTex PPy-coated PET fabric which was prepared similarly as described previously in $[11,12]$, with raw chemicals purchased from Sigma-Aldrich and used without further purification. Stochiometric molar ratio of organic acid dopant, anthraquinone-2-sulfonic acid to pyrrole-monomer (i.e., 0.33:1) was used to ensure complete doping level. The molar ratio of polymerisation catalyst, iron (III) nitrate, to monomer (pyrrole) equal to $2.3 \mathrm{~mol} /$ mol was used for all reactions. The macroscopic texture of this fabric is shown in Figure 1: it is a net with a structure quite useful for application in heating systems, as we shall demonstrate in Section 4 of this paper.

Simultaneous in-situ polymerization and deposition of conductive polypyrrole leads to production of conductive, smooth and uniform coating with thickness under $1 \mu \mathrm{m}$, according to transmission electronic microscope measurements (see Figure 2). As observed in Ref. 13, it is possible a formation of insoluble polymers in the bulk

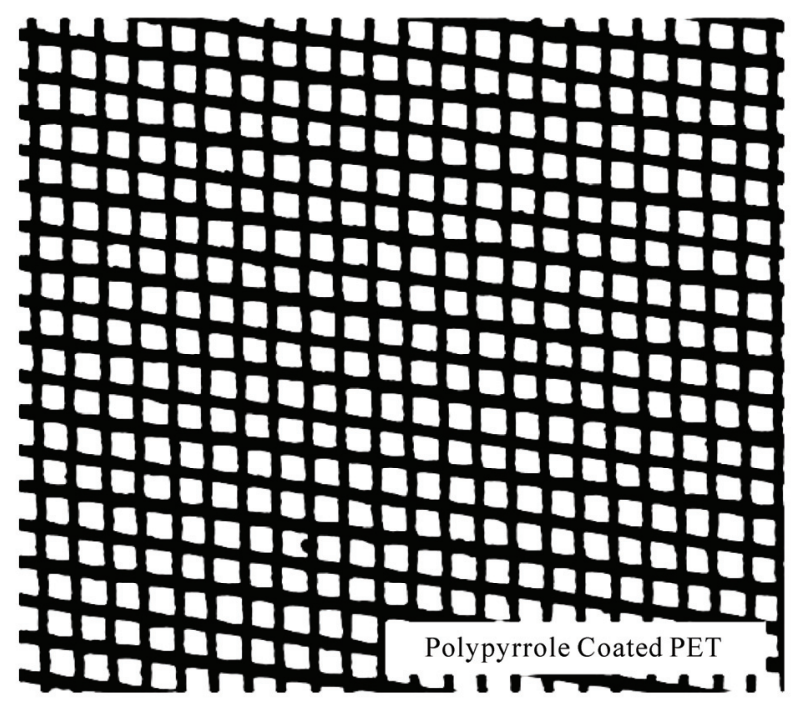

Figure 1. Polypyrrole coated PET net. The image sizes are $4.3 \mathrm{~cm} \times 5 \mathrm{~cm}$.

solution and on the surface of the substrate simultaneously. The bulk polymerization produces dendritic polymer particles in the solution and the surface polymerization forms a polymer film on the substrate surface. Some of the bulk polymer precipitates on the surface of the substrate and then the SEM analysis shows these particles on the fibers.

Figures 3 and 4 are reproducing scanning electron microscope SEM images of PPy coated fibers in a nonwoven textile sample and in a twill textile sample, respectively. These materials possess a good conductivity and then are quite useful for electromagnetic shielding applications, as reported in Ref. 5 and 6. In that paper, the performances of PPy coated fabrics were compared with that of a leno nylon fabric, metalized with a $\mathrm{Ni} / \mathrm{Ag}$ coating.

In the following section we will discuss the Seebeck effect of the PPy-coated net shown in Figure 1 and of the leno Ni/Ag metalized textile, for comparison. For what concerns the heat generation, this will be discussed in Section 4 for the PPy coated net. The metalized leno sample is not useful due to its low resistance. In fact, the PPy/PET net has a DC surface resistivity of $306.0 \Omega / \mathrm{sq}$, whereas the metalized leno has a resistance of $0.22 \Omega / \mathrm{sq}$. The electrical DC surface resistances were measured by using a four-in-line point probe in combination with computerized Loresta-AP meter from Mitsubishi Petrochemical Co., LTD.

\section{Thermal-Electric Effects}

Researches on organic materials for thermoelectric applications have not been so attractive, probably because of their poor electronic transporting characters, till the 

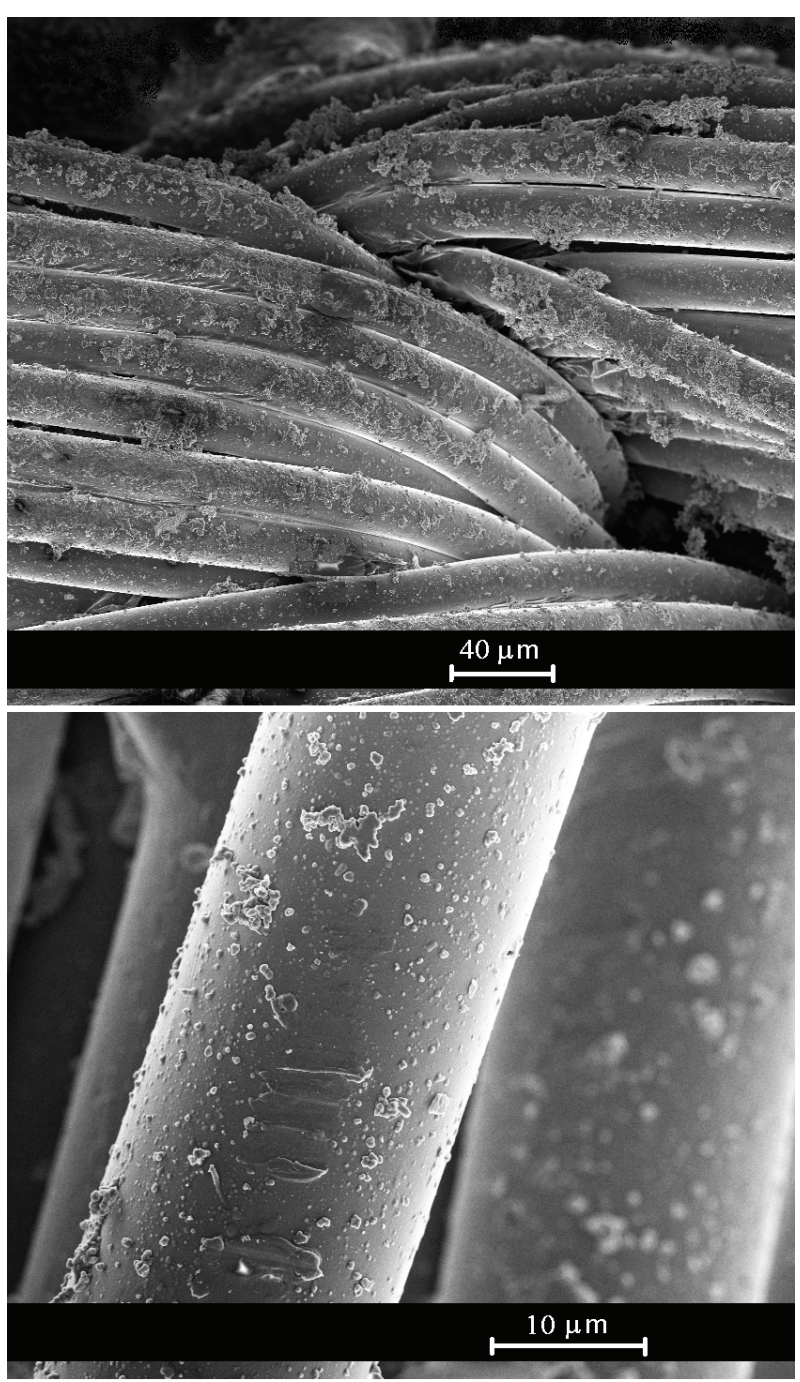

Figure 2. Scanning electron microscope SEM images of the PPy-coated PET net shown in Figure 1. The dust is due to polymer particles in solution, deposited on the fibers of the net. In the lower panel of the image a detail of fibers.

discovery of ICPs. Some of these electrically conducting polymers have gained attention because of their considerable thermal stability. These materials, which are polyaniline and polypyrrole, are considered suitable for applications to electronic devices and sensors [14-17].

For thermal sensors, a systematic investigation on thermoelectric performances of polyaniline [18] and polypyrrole [19] is then interesting. In fact, supposing for polymeric compounds a low thermal conductivity, we can obtain significant advantage of the thermoelectric figure-of-merit. Let us remember that the figure-of-merit is defined as $Z T=\left(S^{2} \sigma / \kappa\right) T$, where $S, \sigma, \kappa$ and $T$ are Seebeck coefficient, electric conductivity, thermal conductivity, and absolute temperature, respectively. The value of $Z T$ for polypyrrole is comparable with that of

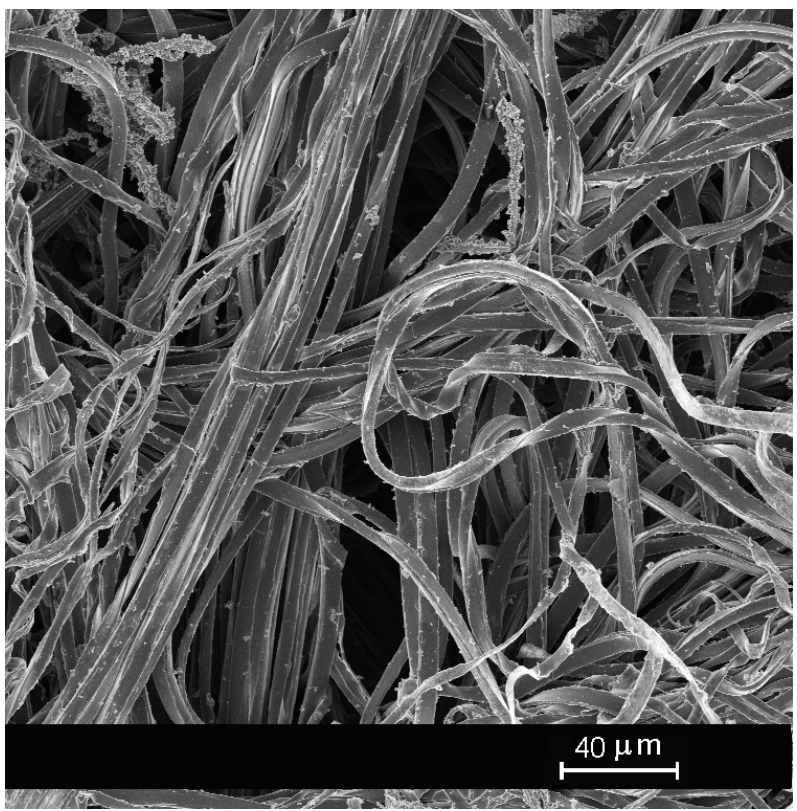

Figure 3. SEM images of PPy coated fibers in a nonwoven textile sample.

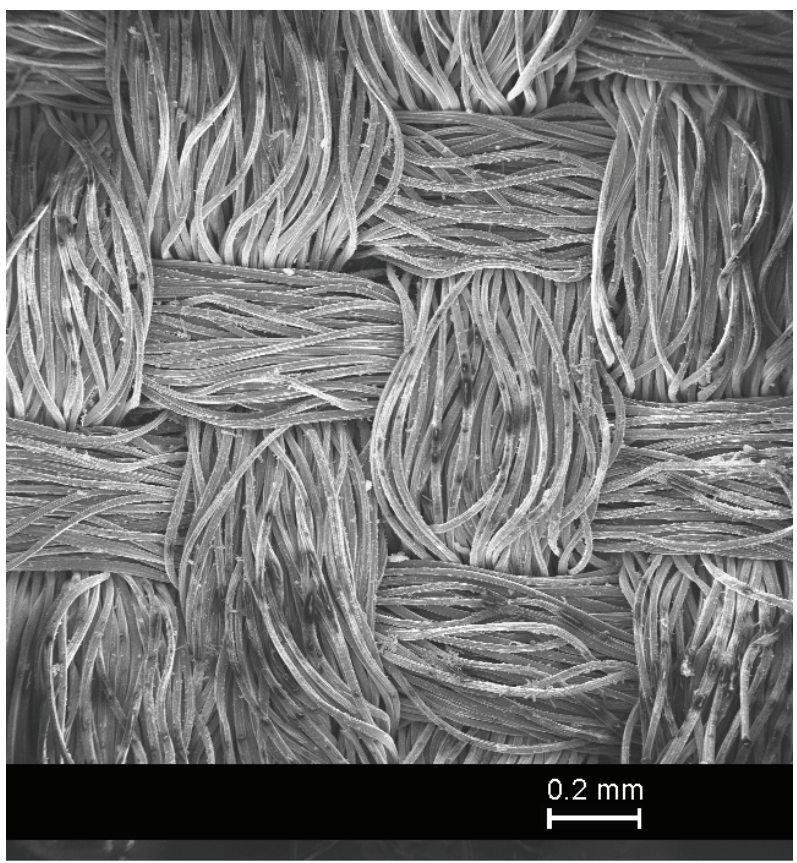

Figure 4. SEM images of PPy coated fibers in a twill textile sample.

some traditionally used inorganic thermoelectric materials $[8,16]$. Besides polyaniline and polypyrrole, other materials, such as polythiophene has been recently investtigated, in the form of nanofilms too [20-21].

We investigated the behavior of the electrical resistiveity as a function of temperature. Starting from room temperature, the resistance of a PPy/PET sample placed in a 
thermostage, was checked till a temperature of $70^{\circ} \mathrm{C}$. The resistance behavior with temperature is typical of a semiconductor with the resistance decreasing linearly as the temperature increases. At room temperature, the resistance was of $172 \Omega$ in a sample with a length of $4 \mathrm{~cm}$, composed by 10 yarns, each yarn with a diameter of 0.05 $\mathrm{cm}$. At $70^{\circ} \mathrm{C}$, the resistance of the sample was of $145 \Omega$. Assuming as in Ref. 23 a thickness in polypyrrole coating of around $1 \mu \mathrm{m}$, the electric conductivity $\sigma$ turns out to be $10^{4} \Omega^{-1} \mathrm{~m}^{-1}$. This estimation of the electrical conduc- tivity of PPy coating is in good agreement with the value of $1.7 \times 10^{4} \Omega^{-1} \mathrm{~m}^{-1}$, given in Ref. 17 .

Thermoelectric Seebeck coefficient $(S)$ and its temperature dependence were determined by connecting a stripe of PPy/PET net $0.5 \mathrm{~cm}$ wide with a copper wire. The two materials are electrically connected by the pressure of a very small silver clip, insulated from the junction. The hot junction was placed in the thermostage with a reference Chromel/Alumel thermocouple and the cold junction between the PPy/PET stripe and copper was thermally anchored at room temperature $\left(26^{\circ} \mathrm{C}\right)$. The same anchoring was used for the cold junction of the Chromel-Alumel thermocouple (a diagram of the experimenttal set-up in Figure 5). Variations in monitored room temperature during measurements were negligible (around $5 \%$ ).

In Figure 6, the behavior of the electro-motive force measured for two such PPy/Copper thermocouples is given as a function of temperature difference $\Delta T$ between the actual hot junction temperature and the room temperature. Assuming a value of Copper e.m.f. vs. Platinum of $0.0076 \mathrm{mV} / \mathrm{K}$ [24], we can estimate a value of $0.0133 \mathrm{mV} / \mathrm{K}$ for the PPy vs. Platinum e.m.f. and positive.

To obtain the figure-of-merit ( $Z T$ ), we estimate the PPy thermal conductivity in the following manner. Thermal diffusivity of PPy films was deduced from measurements by the laser flash method [25]. In Ref. 25, PPy films exhibit a thermal diffusivity ranging from 4 to 8. $\times 10^{-7} \mathrm{~m}^{2} \mathrm{~s}^{-1}$ at room temperature (even lower than the value of $1.3 \times 10^{-6} \mathrm{~m}^{2} \mathrm{~s}^{-1}$ measured for polyaniline [26]). The very low thermal diffusivity of polypyrrole films is originated in the lattice structure, in particular from a dominant amorphous character of the chain structure. An amorphous structure strongly reduces the thermal phonon transport, because strong phonon scattering mechanisms appear [27,28].

We can then assume a value of thermal diffusivity $\alpha$ of about $5 . \times 10^{-7} \mathrm{~m}^{2} \mathrm{~s}^{-1}$ and a specific heat capacity $c=0.4 \mathrm{~J} \mathrm{~g}^{-1} \mathrm{~K}^{-1}$, in the range of the values for organic polymers [26]. The thermal conductivity is then given by $\kappa=\alpha c \rho$. With a density $\rho$ of $1.3 \times 10^{3} \mathrm{~kg} / \mathrm{m}^{3}$ [29], the thermal conductivity of polypyrrole film turns out to

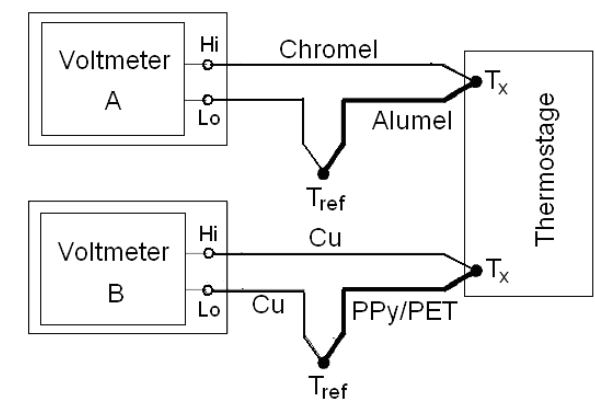

Figure 5. Diagram of the experimental set-up for measuring the Seebeck effect. The electromotive force of two thermocouples are compared: a thermocouple is used as a reference to determine the temperature in the thermostage, the other to determine the unknown electromotive force of the material.

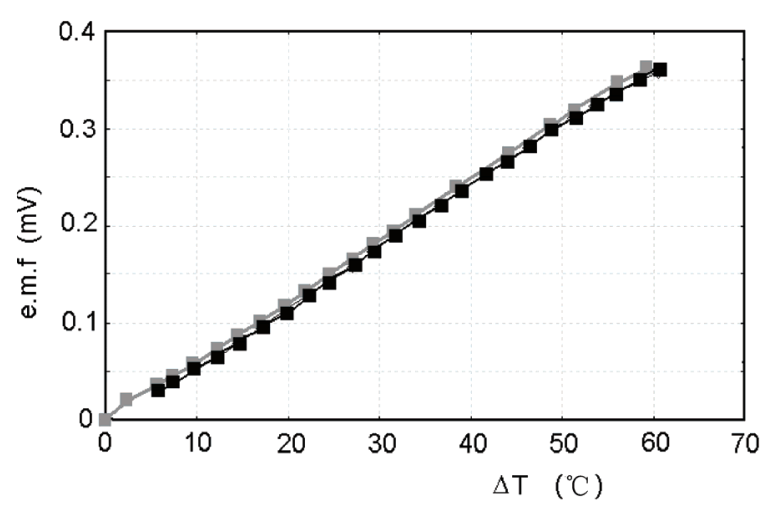

Figure 6. Electromotive force measured for a PPy-PET/ Copper thermocouple as a function of the temperature difference $\Delta T$ between the actual hot junction temperature and the room temperature. The figure reports two diffeent scanning in temperature.

be $0.3 \mathrm{~W} \mathrm{~m}^{-1} \mathrm{~K}^{-1}$. This low thermal conductivity is at least in one order of magnitude lower than that of the best inorganic thermoelectric materials. Using $\sigma=10^{4} \Omega^{-1} \mathrm{~m}^{-1}$, $\kappa=0.3 \mathrm{~W} \mathrm{~m}^{-1} \mathrm{~K}^{-1}$ and our estimate of Seebeck coefficient, we obtain $Z T=1.5 \times 10^{-3}$ at $T=300 \mathrm{~K}$ in agreement with data of polypyrrole films [17].

The power factor $S^{2} \sigma$ is approximately $2 . \times 10^{-6} \mathrm{~W}$ $\mathrm{m}^{-1} \mathrm{~K}^{-2}$. This power factor is better than those reported for polypyrrole in Ref. 30.

Note from Figure 6 the linear behaviour of the thermoelectric power with respect to temperature: this behaviour has been observed in highly doped polyacetylene and in some cases for PANi [30].

Investigations of the electromotive forces with nonwoven and twill samples of Figures $\mathbf{3}$ and $\mathbf{4}$ were also performed, but in this case, the results of measurements are questionable due to a strong dependence on the contact between textile and wires. The best result we obtained was 
of $0.008 \mathrm{mV} / \mathrm{K}$ for the non-woven sample, with a linear behavior with temperature. The nonwoven sample had a DC surface resistivity of $30.0 \Omega /$ sq: we could estimate a quite optimistic value of the electric conductivity $\sigma$ of $10^{5} \Omega^{-1} \mathrm{~m}^{-1}$. Assuming the value $0.005 \mathrm{mV} / \mathrm{K}$ as a possible estimate of Seebeck coefficient, we could reach a power factor $S^{2} \sigma$ of $0.5 \times 10^{-5} \mathrm{~W} \mathrm{~m}^{-1} \mathrm{~K}^{-2}$, and a figure-of-merit $Z T=4.8 \times 10^{-3}$ at a temperature of $T=300 \mathrm{~K}$.

The Seebeck electromotive force of the nylon leno sample, coated $\mathrm{Ni} / \mathrm{Ag}$, connected with $\mathrm{Cu}$ was also measured and the behavior is shown in Figure 7 (curve b). The same figure shows that a thermocouple built with $\mathrm{Ni} / \mathrm{Ag} / \mathrm{Nylon}$ and PPy/PET can give the higher electromotive force (curve a). We have also prepared a thermocouple with PPy/PET and a yarn composed of comercial carbon fibers (curve c).

As shown by the measurements here reported, PPy coating can be successfully used with other conductive yarns, for instance Copper, $\mathrm{Ni} / \mathrm{Ag}$ coated yarns or carbon fibers, to obtain stable textile thermopiles, which are able to provide thermo-powers as typical semiconductors. The proposal of use coated fabrics in thermal-electric devices is not new [31]. Let us remark that we used a commercial PPy-coated textile, with a stable coating, not an ad-hoc prepared film, and this, in our opinion, is relevant for industrial applications.

\section{Heat Generation from Fabrics}

A possible application of the PPy-coated net, due to its fabric structure, is in heating devices. The following setup for detecting heat generation is used [32,33]. This set-up was previously developed to study polypyrrole samples prepared in Biella laboratory for innovative textiles. Those samples were too dusty to allow them to be used in applications. An adjustable Variac power supply was used to generate an AC current/voltage over the fabric. Voltage and current were monitored by Keithley voltmeter and amperometer. A square shape fabric $(6 \mathrm{~cm}$ $\times 6 \mathrm{~cm}$ ) was positioned between two pressed electric contacts (a diagram of the arrangement in Figure 8). The temperature rise was measured using an Omega infrared thermometer, placed to control the center of the sample.

In Figure 9, the behavior of the temperature as a function of the current is given, with the rise of the voltage. According to the power law, the maximum theoretical power achieved from the fabrics is: $P=V I$, where $P$ is the power developed and $V, I$ the voltage and current. The $\mathrm{AC}$ current frequency is $50 \mathrm{~Hz}$. In Figure 10, the power and the impedance as a function of current are shown.

In Ref. 12, the power density per unit area is assumed to be: $P=V^{2} / R_{S} l^{2}$, where $R_{S}$ is the surface resistance and $l$ the size of the sample. Our highest value is

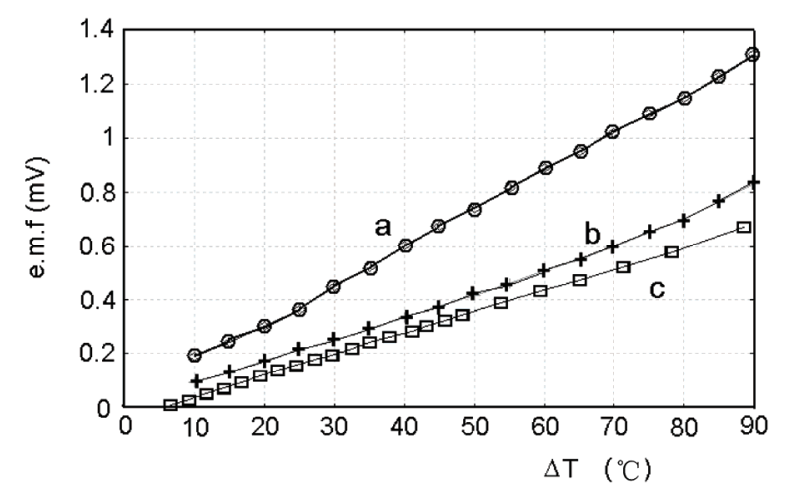

Figure 7. Electromotive force measured for Ni/Ag/Nylon/ Contex. (a) for Ni/Ag/Nylon/Copper, (b) and for PPy/PET/ Carbon Fibers; (c) as a function of the temperature difference $\Delta T$ between the actual hot junction temperature and cold junction at room temperature.

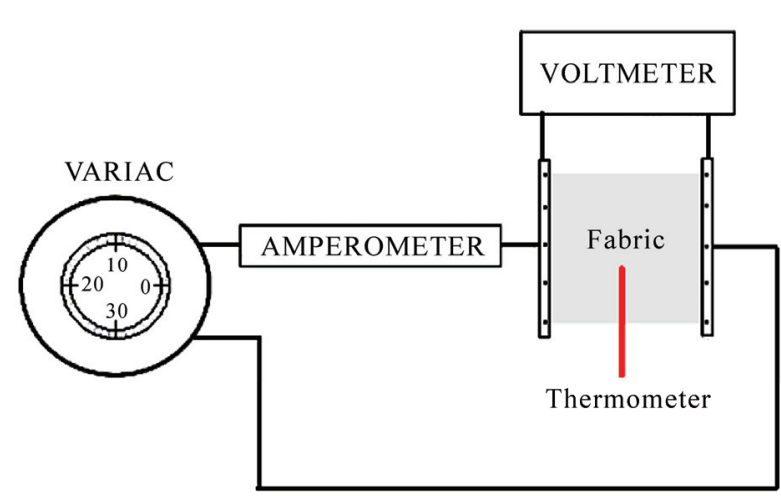

Figure 8. Experimental set-up diagram for measuring the heating effect of a textile. Current and voltage across the sample must be monitored.

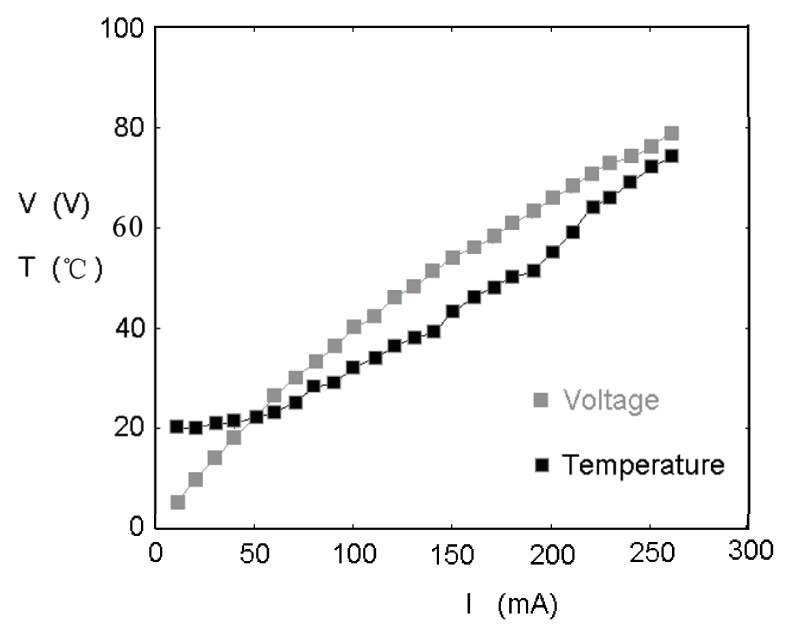

Figure 9. Behavior of the voltage and temperature of the PPy coated sample as a function of the current, measured with voltmeter and thermometer. 


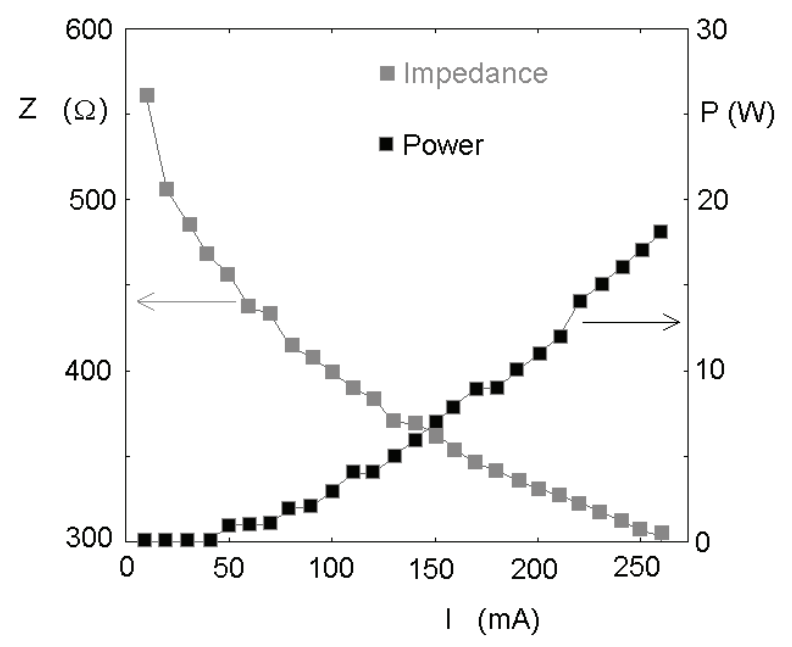

Figure 10. Behavior of the impedance and power developed by the PPy coated sample as a function of the current. The values of impedance and power are estimated from data of Figure 8.

$370 \mathrm{~W} / \mathrm{m}^{2}$ in agreement with the value obtained in Ref. 12.

\section{Conclusions}

The conversion electricity-heat has always attracted a great attention because of applications in heaters, coolers and thermoelectric power generators. The parameter measuring the suitability of a material for these applications is the figure-of-merit. In order to have high values of the figure-of-merit, a material must have high charge transport conductivity, high Seebeck coefficient and low thermal conductivity. The intrinsically conducting polymers can be considered as a new generation of thermoelectric materials, due to their characteristic that often are achieving a figure-of-merit comparable with that of typical semiconductors. Other attractive features are the low cost of material resources, an easy synthesis and processing into desired forms.

Many conducting polymers have been investigated as thermal materials, among them polyaniline, polythiophenes and polypyrroles. Here we have studied the properties of a commercially available polypyrrole coated fabric. As shown by measurements, a Seebeck effect can be achieved by using a PPy conducting coating of a PET fabric. According to the Kelvin relation between Seebeck $S$ and Peltier $\Pi$ coefficients, $\Pi=S T$, we can also imagine a possible application in cooling devices of polypyrrole coated fabrics.

We have also seen that with PPy/PET fabrics, it is possible to easily make heating fabrics. Since the coating with polypyrrole is possible on many different fibers [34], the potential applications of polypyrrole in the building of heating pads is relevant. We suggest then that PPycoated fabrics may be practically useful for many applications, including flexible, portable surface-heating elements for medical or other applications.

\section{Acknowledgements}

Authors thank Angelica Chiodoni for SEM analysis.

\section{REFERENCES}

[1] Y. Cao, P. Smith and A. J. Heeger, "Counter-Ion Induced Processibility of Conducting Polyaniline," Synthetic Metals, Vol. 57, No. 1, 1993, pp. 3514-3519.

[2] C. K. Chiang, C. R. Fincher, Y. W. Park, A. J. Heeger, H. Shirakawa, E. J. Louis, S. C. Gau and A. G. MacDiarmid, "Electrical Conductivity in Doped Polyacetylene," Physics Review Letters, Vol. 39, No. 17, 1997, pp. 1098$1101 .$.

[3] A. G. MacDiarmid, "Polyaniline and Polypyrrole: Where are We Headed?" Synthetic Metals, Vol. 84, No. 1-3, 1997, pp. 27-34.

[4] J. Edenbaum, Ed., "Plastics Additives and Modifiers Handbook," Van Nostrand Reinhold, New York, 1992.

[5] J. Avloni, R. Lau, M. Ouyang, L. Florio, A. R. Henn and A. Sparavigna, "Shielding Effectiveness Evaluation of Metallized and Polypyrrole-Coated Fabrics," Journal of Thermoplastic Composite Materials, Vol. 20, No. 3, 2007, pp. 241-254.

[6] J. Avloni, M. Ouyang, L. Florio, A. R. Henn and A. Sparavigna, "Polypyrrole-Coated Nonwovens for Electromagnetic Shielding," Journal of Industrial Textiles, Vol. 38, No. 3, 2008, pp. 55-68.

[7] A. R. Henn and R. M. Cribb, "Modelling the Shielding Effectiveness of Metallized Fabrics," Interference Technology Engineering Master (ITEM) Update, 1993, pp. 49-57.

[8] Y. Y. Wang and X. L. Jing, "Intrinsically Conducting Polymers for Electromagnetic Interference Shielding," Polymers for Advanced Technologies, Vol. 16, No. 4, 2005, pp. 344-351.

[9] Milliken and Co., Milliken. http://www.milliken.com

[10] A. F. Ioffe, "Semiconductor Thermoelements and Thermoelectric Cooling," Infosearch, London, 1957.

[11] H. H. Kuhn and A. D. Child, "Handbook of Conducting Polymers," Marcel Dekker, New York, 1998.

[12] H. H. Kuhn, A. D. Child and W. C. Kimbrell, "Toward Real Applications of conductive Polymers, Synthetic Metals," Vol. 71, No. 1-3, 1995, pp. 2139-2142.

[13] E. Hakansson, A. Kaynak, T. Lin, S. Nahavandi, T. Jones and E. Hu, "Characterization of Conducting Polymer Coated Synthetic Fabrics for Heat Generation," Synthetic Metals, Vol. 144, No. 1, 2004, pp. 21-28.

[14] D. Kumar and R. C. Sharma, "Advances in Conductive Polymers," European Polymer Journal, Vol. 34, No. 8, 1998, pp. 1053-1060.

[15] D. Braun, "Semiconducting polymer LEDs," Materials Today, Vol. 5, No. 6, 2002, pp. 32-39. 
[16] J. V. Hatfield, P. Neaves, K. Persaud and P. Travers, "Towards an Integrated Electronic Nose Using Conducting Polymer Sensors," Sensors and Actuators B, Vol. 18, No. 1-3, 1994, pp. 221-228.

[17] H. Yan, T. Ishida and N. Toshima, "Thermoelectric Properties of Electrically Conductive Polypyrrole Film, Thermoelectrics," Proceedings ICT 2001, XX International Conference on Thermoelectrics, Beijing, 2001, pp. 310313.

[18] F. Yakuphanoglu and B. F. Şenkal, "Electronic and Thermoelectric Properties of Polyaniline Organic Semiconductor and Electrical Characterization of Al/PANI MIS Diode," Journal of Physical Chemistry C, Vol. 111, No. 4, 2007, pp. 1840-1846.

[19] A. B. Kaiser, O. Mercier, H. J. Trodahl, N. T. Kemp, C. J. Liu, B. Chapman, A. M. Carr, R. G. Buckley, A. C. Partridge, J. Y. Lee, C. Y. Kim, A. Bartl, L. Dunsch, W. T. Smith and J. S. Shapiro, "Thermoelectric Power and Conductivity of Different Types of Polypyrrole," Journal of Polymer Science: Part B: Polymer Physics, Vol. 37, No. 9, 1999, pp. 953-960.

[20] M. Scholdt, H. Do, J. Lang, A. Gall, A. Colsmann, U. Lemmer, J. D. Koenig, M. Winkler and H. Boettner, "Organic Semiconductors for Thermoelectric Applications," Journal of Electronic Materials, Springer, Boston, 2010.

[21] Y. Shinohara, K. Hiraishi, H. Nakanishi, Y. Isoda and Y. Imai, "Study on Thermoelectric Properties of Conductive Polymers," Transactions on Materials Research Society of Japanese, Vol. 30, No. 4, 2005, pp. 963-969.

[22] B. Y. Lu, C. C. Liu, S. Lu, J. K. Xu, F. X. Jiang, Y. Z. Li and Z. Zhang, "Thermoelectric Performances of Freestanding Polythiophene and Poly(3-Methylthiophene) Nanofilms," Chinese Physics Letters, Vol. 27, No. 5, 2010, pp. 057201.1-057201.4.

[23] A. R. Henn, "Calculating the Surface Resistivity of Conductive Fabrics," Interference Technology Engineering Master (ITEM) Update, 1996, pp. 66-72.

[24] D. E. Gray, "American Institute of Physics Handbook," McGraw-Hill, New York, 1963.

[25] M. Y. Lim, W. M. M. Yunus, A. Kassim and H. N. M. E.
Mahmud, "Photoacoustic Measurement of Thermal Diffusivity of Polypyrrole Conducting Polymer Composite Films," American Journal of Applied Sciences, Vol. 6, No. 2, 2009, pp. 313-316.

[26] H. Yan, N. Sada and N. Toshima, "Thermal Transporting Properties of Electrically Conductive Polyaniline Films as Organic Thermoelectric Materials," Journal of Thermal Analysis and Calorimetry, Vol. 69, No. 3, 1992, pp. 881887.

[27] M. Omini and A. Sparavigna, "Role of Grain Boundaries as Phonon Diffraction Gratings in the Theory of Thermal Conductivity," Physics Review B, Vol. 61, No. 10, 2000, pp. 6677-6688.

[28] A. Sparavigna, M. Omini, A. Pasquarelli and A. Strigazzi, "Thermal Diffusivity and Conductivity in Low-Conducting Materials: A New Technique," International Journal of Thermophysics, Vol. 13, No. 2, 1992, pp. 351360.

[29] J. Tietje-Girault, C. Ponce de León and F. C. Walsh, "Electrochemically Deposited Polypyrrole Films and their Characterization," Surface \& Coatings Technology, Vol. 201, No. 12, 2007, pp. 6025-6034.

[30] A. Shakouri and S. Li, "Thermoelectric Power Factor for Electrically Conductive Polymers," Proceedings of International Conference on Thermoelectrics, Baltimore, September 1999.

[31] E. Hu, A. Kaynak and Y. Li, "Development of a Cooling Fabric from Conducting Polymer Coated Fibres: Proof of Concept," Synthetic Metals, Vol. 150, No. 2, 2005, pp. 139-143.

[32] L. Florio, "Dissertation for Master Degree, Studio dei Polimeri Intrinsecamente Conduttori: Applicazione al tessile Elettronico (in Italian)," Politecnico di Torino, 2004.

[33] L. Florio and A. Sparavigna, "Thermoelectric Properties of Conducting Polymers," INF Meeting, National Conference on the Physics of Matter, Genova, 2004.

[34] D. T. Seshadri and N. V. Bhat, "Synthesis and Properties of Cotton Fabrics Modified with Polypyrrole," Sen'I Gakkaishi, Vol. 61, No. 4, 2005, pp. 103-108. 